\title{
16. A LIBRARY OF STANDARD PROGRAMMES FOR CONSTRUCTING NUMERICAL THEORIES FOR STUDYING THE MOTION AND EVOLUTION OF THE ORBITS OF THE MINOR BODIES OF THE SOLAR SYSTEM
}

\author{
N. A. BOKHAN \\ Institute for Theoretical Astronomy, Leningrad, U.S.S.R.
}

\begin{abstract}
At the Institute for Theoretical Astronomy we have formed a library containing about 120 standard computer programmes. They include calculation of rectangular coordinates and velocities from elements, Lagrangian interpolation, and orbit improvement by the Eckert-Brouwer method. For the investigation of the motions of the minor bodies of the solar system we have constructed a programme for integration with a variable step and making allowance for perturbations by all the major planets and for nongravitational effects. The calculation of the perturbations is carried out using Herrick's vector parameters by the method of variation of arbitrary constants. The programme has been used for studying the motion of $\mathrm{P} /$ Encke.
\end{abstract}

\section{The Library of Standard Programmes}

When solving astronomical problems that require cumbersome computations it is important to proceed systematically. Consequently, over the years various tables have been made, as the tools of computation have advanced from logarithms to desk calculators. Because of primitive means of computing, the solution of complicated problems in the past took great amounts of time: Encke spent 40 years investigating the motion of the comet named after him; and Backlund studied the same comet for 38 years. Desk calculators considerably facilitated and accelerated the computation process.

The advent of electronic computers in the 1950's resulted in drastic changes: it became possible to pose and solve astronomical problems which were almost insoluble with desk calculators, and all the more so with logarithms. Increasingly difficult problems require more time spent on preparing programmes for the computer. As a result, it is necessary to simplify programming, to use the computer systematically and, particularly, to spend less time debugging the programme.

There are two possibilities for accomplishing this:

(1) writing the programme in ALGOL; (2) forming a library of standard astronomical programmes, as is done for mathematical problems.

When constructing numerical theories for the motion and investigation of the evolution of orbits of the minor bodies of the solar system with the use of computers, it is necessary to have a complex set of programmes. A considerable part of the computer's memory is used for the coordinates of the major planets and other constants. The memory of the computer is used more rationally when programmes are written in machine language rather than in the ALGOL. That is why we have pre- 
ferred to follow the second possibility above and provide a library of standard programmes.

Rather than store tables of various functions in the computer's memory it is convenient to have special programmes that compute values of these functions according to particular algorithms. These auxiliary programmes are called standard programmes (SP), because they are constructed so as to make them suitable for use in any other programme and with any values of the arguments within the limits given in the instructions for their use. For every computer there is a set of SP's for the calculation of elementary functions and many of the methods of linear algebra, mathematical analysis, and probability theory.

The library set up at the Institute for Theoretical Astronomy has SP's for many common astronomical calculations, such as the solution of Kepler's equation, calculation of rectangular coordinates and velocities from elements, calculation of the coordinates of the Sun from Newcomb's theory, interpolation in tables by the Lagrange method (with a fixed and variable step). More complicated SP's, such as comparison of the observed and computed coordinates of any object, have been constructed by adding to the simple programmes already prepared.

The use of the library of SP's for the solution of complicated astronomical problems with a computer permits economical employment of the computer's memory, because the SP's required at a given stage of calculation are merely brought in as needed.

The library at the Institute contains about 120 SP's. The author has composed more than 50 of them. Together with Makover she has constructed a set of programmes for the improvement of elliptical orbits with six as well as eight unknowns.

The following files may be considered as necessary supplements to the SP's:

(1) rectangular coordinates of the major planets Venus-Pluto at 20-day intervals with allowance for all mutual perturbations during 1660-2060 (Kazimirchak-Polonskaya);

(2) coordinates of Mercury 1900-2000 at 5-day intervals, using the tables by Duncombe et al. (1965), with the coordinates thoroughly checked and stored on punched cards and magnetic tape (Bokhan);

(3) coordinates of observatories on punched cards (Belyaev and Bokhan).

There are many methods for the numerical integration of differential equations, such as those of Encke, Cowell, Numerov, and Subbotin. Perturbations for any object can be computed and also checked by using two or more such methods each of which has advantages and disadvantages. At the Institute standard programmes have been constructed for integration using various methods:

(1) integration with double precision, automatic change of step, allowance for perturbations by all major planets and nongravitational effects (KazimirchakPolonskaya);

(2) integration by Cowell's method with automatic selection of step and consideration of perturbations by all major planets (Belyaev);

(3) integration of $n$ bodies by Myachin's method (Sizova);

(4) integration with a variable step and consideration of perturbations by all 
major planets and of nongravitational effects by the method of variation of arbitrary constants using Herrick's vector parameters (Bokhan).

This is all part of a general programme which includes the simultaneous comparison with observations and the computation of ephemerides.

\section{Numerical Integration Programme}

With the development of modern electronic computers significant changes have been made in the classical methods of the variation of arbitrary constants. To make the process more suitable and systematic for programming, vector elements were used. Introduced by Milankovich (1941), vector elements were developed by Bilimovich (1943) and Popovich (1950). Such methods were not popular until computers were used for astronomical calculations.

Herrick (1948) and Musen (1954) obtained equations for the perturbations of vector elements in a form suitable for computers. Herrick (1953) applied his method to the minor planet Icarus. Vector elements can be successfully used for perturbations on comets of large or moderate orbital eccentricity; the method is especially convenient for objects of small perihelion distance, when Cowell's method requires an extremely small integration step.

The method of quadratures has been used for calculating the perturbations from derivatives, differences up to the fourth order being considered. For automatic selection of the integration interval Kazimirchak-Polonskaya's (1967) criterion was used, depending on the distance from the Sun and perturbing planets.

Nongravitational effects were considered on the assumption that the secular acceleration is in the form of an impulse acting exclusively at perihelion (Makover, 1955). Let $t_{0}$ be the osculation epoch and $t$ a given moment of time. If a comet passes through perihelion at time $t^{\prime}$ in the interval $t_{0}-t$, we can write

$$
\begin{aligned}
M & =M_{0}+\mu_{0}\left(t-t_{0}\right)+\mu^{\prime}\left(t-t^{\prime}\right)+\text { perturbations } \\
\mu & =\mu_{0}+\mu^{\prime}+\text { perturbations } \\
\varphi & =\varphi_{0}+\varphi^{\prime}+\text { perturbations, }
\end{aligned}
$$

where $\mu^{\prime}=2 \kappa / P, P$ is the period of a comet (in days), and $\mu^{\prime}$ and $\varphi^{\prime}$ are the secular variations in the mean daily motion and eccentric angle.

The programme described above has been used for the integration of the equations of motion of P/Encke during 1967-1971, using a variable step and taking into account perturbations by the eight major planets Mercury-Neptune and nongravitational effects. A search ephemeris was calculated for 1970-1971 (Bokhan, 1970).

During the next five years we intend to study the motion of $P /$ Encke over the total interval since discovery. We have started by considering the little-studied period 1911-1921, but the results obtained up to now are preliminary and hence not published.

\section{Acknowledgments}

In conclusion I wish to express my cordial thanks to E. I. Kazimirchak-Polonskaya 
for her constant attention to this work, her valuable comments, for supplying her standard programmes, and for check calculations by her method and integration programme. I am equally grateful to N. A. Belyaev for supplying programmes and for his active help in the investigation. Others who participated in setting up the programme library were S. G. Makover, N. S. Kochina, L. E. Nikonova, V. A. Izvekov, M. Ya. Shmakova, T. I. Podunova and T. K. Nikol'skaya.

\section{References}

Bilimovich, A.: 1943, Astron. Nachr. 273, 161.

Bokhan, N. A.: 1970, Kiev. Komet. Tsirk. No. 103.

Duncombe, R. L., Tufekcioglu, Z., and Larson, G.: 1965, U.S. Naval Obs. Circ. No. 106.

Herrick, S.: 1948, Publ. Astron. Soc. Pacific 60, 321.

Herrick, S.: 1953, Astron. J. 58, 156.

Kazimirchak-Polonskaya, E. I.: 1967, Trudy Inst. Teor. Astron. 12, 24.

Makover, S. G.: 1955, Trudy Inst. Teor. Astron. 4, 133.

Milankovich, M.: 1941, Kanon der Erdbestrahlung, Belgrade, p. 78.

Musen, P.: 1954, Astron. J. 59, 262.

Popovich, B.: 1950, Compt. Rend. Belgrade 198, 129.

\section{Discussion}

G. Sitarski: It is particularly difficult to link two apparitions of a comet separated by a close approach to Jupiter and absolutely necessary to allow for perturbations in the calculation of the differential coefficients. Do you allow for these?

N. A. Bokhan: Not yet, but work is in progress on this problem. 